Relations industrielles

Industrial Relations

\title{
Claude Gervais, Comprendre et prévenir le burnout
}

\section{Denis Morin}

Volume 47, numéro 1, 1992

URI : https://id.erudit.org/iderudit/050760ar

DOI : https://doi.org/10.7202/050760ar

Aller au sommaire du numéro

Éditeur(s)

Département des relations industrielles de l'Université Laval

ISSN

0034-379X (imprimé)

1703-8138 (numérique)

Découvrir la revue

Citer ce compte rendu

Morin, D. (1992). Compte rendu de [Claude Gervais, Comprendre et prévenir le burnout]. Relations industrielles / Industrial Relations, 47(1), 181-182.

https://doi.org/10.7202/050760ar

Tous droits réservés (C) Département des relations industrielles de l'Université Laval, 1992
Ce document est protégé par la loi sur le droit d'auteur. L'utilisation des services d'Érudit (y compris la reproduction) est assujettie à sa politique d'utilisation que vous pouvez consulter en ligne.

https://apropos.erudit.org/fr/usagers/politique-dutilisation/ 
There is no substantial proof that big powers will be able to impose their own socio-economic schemes on other societies, as long as the latter follow successfully their own models. The study done by G. Esping-Andersen reinforces the pluralist option of the socio-economic development.

Alexander J. Matejko

University of Alberta

Comprendre et próvenir le burnout, par Claude Gervais, Montréal, Les éditions Agence d'Arc, 1990, 214 p., ISBN 2-89022-233-0

Le concept de burnout est apparu au début des années 70 . II tire ses origines des travaux de Freudenberger afin de décrire une combinaison de symptômes qui se manifestent notamment par un épuisement chronique, une fatigue physique, une absence d'implication au travail, une déshumanisation des services aux bénéficiaires ainsi qu'une diminution des réalisations au travail. L'ouvrage de Claude Gervais est articulé autour de la prévention du burnout au travail.

L'auteur développe un modèle diagnostic du burnout qui sert de cadre d'analyse dans l'élaboration d'une démarche préventive. Plus particulièrement, une description ponctuelle des causes, du processus, des conséquences du burnout ainsi que des méthodes d'intervention sont présentées et abondamment illustrées à l'aide d'exemples cliniques. Afin de concrétiser l'orientation résolument clinique du livre, des questionnaires d'évaluation sur la vulnérabilité au burnout et des études de cas accompagnent un ensemble de principes à respecter dans l'élaboration d'un programme de relation d'aide à la victime de burnout.

Le plan de travail qui est à l'origine de ce volume reflète bien la préoccupation précitée. La structure du livre s'organise autour de quatre chapitres. L'introduction établit les bases conceptuelles de la notion de burnout. L'auteur conceptualise le burnout comme un processus cumulatif à 6 étapes et intègre la littérature sur le stress au travail dans son modèle. Par ailleurs, il emprunte certains éléments des modèles de Cherniss, Golembiewski, Maslach et Pines. Le burnout est le résultat d'une accumulation de tensions mal canalisées entre l'individu et son emploi et entraînant une impression d'échec, une baisse d'efficacité ainsi qu'une perte d'intérêt notable pour le travail (p. 22). La définition proposée renvoit à la perspective interactionniste développée par French pour le diagnostic des causes du burnout. Selon l'approche interactionniste, le stress au travail survient quand l'environnement de travail exerce des demandes sur l'individu qui ne cadrent pas avec ce que ce dernier peut offrir. Le burnout constitue l'étape finale à une progression de tentatives infructueuses afin de composer avec des conditions de stress au travail. Plus spécifiquement, lorsque la demande du milieu de travail taxe exagérément la capacité d'adaptation de l'individu, ce dernier est susceptible d'être victime de burnout. La relation entre la demande du milieu de travail et le burnout est donc modérée par le stress au travail. L'auteur souligne également que le support social constitue une variable modératrice importante dans cette relation. 
Les deux chapitres suivants sont consacrés à l'étude des facteurs individuels et organisationnels impliqués dans le développement du burnout afin de pouvoir intervenir à ces deux niveaux. L'auteur attire l'attention sur le rôle de la perception et des différences individuelles dans la genèse du burnout. En effet, les objectifs et les besoins personnels affectent la demande du milieu de travail qui par ricochet agit sur la capacité d'adaptation de l'individu et la vulnérabilité au burnout. La personnalité de l'individu influence également la prédisposition au burnout. Parmi l'ensemble des facteurs individuels énumérés, la personnalité de type A représente le principal facteur à risque élevé. L'individu peut être à toutes fins utiles sa principale cause de burnout.

L'auteur rapporte près de vingt caractéristiques organisationnelles. Parmi celles-ci, on retrouve le manque d'autonomie, les opportunités restreintes d'actualisation de soi au travail, les rétributions limitées ainsi que les conditions physiques de travail. À peu près toutes les composantes organisationnelles vraisemblablement impliquées dans le stress au travail peuvent aussi contribuer au burnout. Devant l'importance des facteurs organisationnels, l'organisation du travail constitue la première cible d'intervention. Ainsi, l'auteur met en évidence une série de solutions possibles afin de corriger les effets néfastes de l'organisation du travail sur la susceptibilité au burnout.

Le quatrième et dernier chapitre porte sur le processus du burnout. L'auteur effectue une analyse minutieuse des comportements types et des mécanismes d'adaptation de l'individu pour l'ensemble des étapes de développement du burnout. Par ailleurs, il y a une discussion des aspects pratiques de l'intervention thérapeutique auprès de la victime du burnout.

Malgré l'intérêt du sujet, cet ouvrage de vulgarisation vise surtout les praticiens et le vaste public. En effet, il n'y a aucune analyse rigoureuse et critique de la littérature théorique et empirique sur le burnout et le stress au travail. Par ailleurs, les références bibliographiques sont inexistantes. Un tel ouvrage ne convient pas au milieu universitaire. Cependant, le volume sera utile aux praticiens qui veulent se familiariser avec la notion de burnout et intervenir à l'intérieur d'une démarche de relation d'aide auprès de la victime de burnout en milieu de travail.

Denis MORIN

Université Laval 